\title{
External quality assessment of urinary methylmalonic acid quantification - results of a pilot study
}

\author{
Michael Vogeser ${ }^{1, *}$, Wilhelm Müller ${ }^{2}$ and \\ Stefan Lorenzl ${ }^{3}$ \\ ${ }^{1}$ Institute of Clinical Chemistry, Hospital of the \\ University of Munich, Munich, Gemany \\ ${ }^{2}$ Chromsystems $\mathrm{GmbH}$, Munich, Germany \\ ${ }^{3}$ Clinic of Neurology, Hospital of the University of \\ Munich, Munich, Germany
}

Keywords: cobalamin deficiency; EQA; methylmalonic acid; urine.

Measurement of methylmalonic acid (MMA) is recognised as a useful tool in the diagnostic work-up of suspected cobalamin deficiency (1). Increased concentrations of MMA in both serum and urine are indicative of cobalamin deficiency at the tissue level. MMA measurement in serum as the sample material is convenient from a logistic point of view, since analysis can be performed using residual sample material after serum cobalamin measurement requested as a screening test. The major drawbacks of serum MMA measurement, however, are the impact of impaired renal function leading to high MMA concentrations, irrespective of individual cobalamin status (2), and the typically very low concentration range of MMA in serum samples. In urine, far higher concentrations of MMA are present compared to serum, facilitating analyses. Furthermore, calculation of the MMA/creatinine ratio can compensate impaired MMA excretion in renal failure.

While external quality assessment programs for serum MMA quantification have been implemented (3), no corresponding services are available for urinary MMA measurement so far, and no commercial materials for assay calibration and quality control are available. In the January 2006 issue of Clinical Chemistry and Laboratory Medicine, we announced a pilot study on external quality assessment of urinary MMA measurement and invited laboratories to participate in this investigation. In response to this announcement, a total of ten European laboratories requested test samples, and lyophilised aliquots of four study samples were shipped. These samples were manufactured (Chromsystems $\mathrm{GmbH}$, Munich, Germany) by spiking a native urine sample from a healthy volunteer (sample B) with different quantities of a pure

*Corresponding author: Michael Vogeser, Institute of Clinical Chemistry, Hospital of the University of Munich, Marchioninistr. 15, 81377 Munich, Germany

Phone: +49-89-70953221, Fax: +49-89-70953240,

E-mail: michael.vogeser@med.uni-muenchen.de aqueous solution of methylmalonic acid (Aldrich, Taufkirchen, Germany; catalogue no. M5,405-8, Lot 32174-494); the calculated increase in MMA concentrations in the spiked samples was $+3 \mathrm{mg} / \mathrm{L}$ (sample C), $+9 \mathrm{mg} / \mathrm{L}$ (sample A), and $+20 \mathrm{mg} / \mathrm{L}$ (sample D). The creatinine concentration of the samples was $0.57 \mathrm{~g} / \mathrm{L}$ (Jaffe).

Results expressed as mmol MMA per mol creatinine were reported up to June 2006 by the participants (conversion of units: $[\mathrm{mmol} / \mathrm{mol}$ creatinine $] \times 1.04 \rightarrow[\mathrm{mg} / \mathrm{g}$ creatinine $]$ ). Analysis of MMA was carried out by gas chromatography-mass spectrometry in nine laboratories, and by liquid chromatography-tandem mass spectrometry in the remaining laboratory.

Mean creatinine-related urinary MMA concentrations reported for the four study samples are given in Table 1, together with the respective inter-laboratory coefficients of variation. All of the results are shown in Figure 1. The upper limit of the reference range for urinary MMA reported in different studies varies from 3.5 to $5.1 \mathrm{mg} / \mathrm{g}$ creatinine (4-7). The intraindividual variation of urinary MMA excretion in non-cobalamindeficient individuals can be substantial, with coefficients of variation of approximately $30 \%$ (8).

For each result reported by the participants - incorporating the inaccuracy of MMA and creatinine quantification - the relative deviation from the mean for all results reported for each sample was calculated. This deviation was $>20 \%$ in 12 of the total 40 measurements $(30 \%)$. Four laboratories reported results with less than $20 \%$ deviation for all four samples; two laboratories reported results with less than $20 \%$ deviation for three of the four samples. Two further laboratories reported results with less than $20 \%$ deviation for two of the four samples. Among the results for all samples, three had a deviation of more than $20 \%$ in one laboratory, and in one laboratory the deviation for all four samples was $>20 \%$.

Our data suggest that in a sample of ten European clinical laboratories, urine with normal MMA concentrations (in relation to preliminary reference ranges)

Table 1 Descriptive statistics for urinary methylmalonic acid (MMA) results for ten laboratories observed for four study samples.

\begin{tabular}{lcccrr}
\hline \multirow{2}{*}{$\begin{array}{l}\text { Sample } \\
\text { ID }\end{array}$} & \multicolumn{2}{l}{ MMA, mmol/mol creatinine } & \multirow{2}{*}{ CV, \% } \\
\cline { 2 - 4 } & Mean & SD & Min & Max & \\
\hline A & 13.0 & 1.2 & 9.6 & 15.5 & 9.1 \\
B & 0.8 & 0.3 & 0.5 & 1.4 & 44.2 \\
C & 5.1 & 1.1 & 2.9 & 6.9 & 21.0 \\
D & 30.0 & 6.0 & 19.2 & 44.7 & 20.1 \\
\hline
\end{tabular}




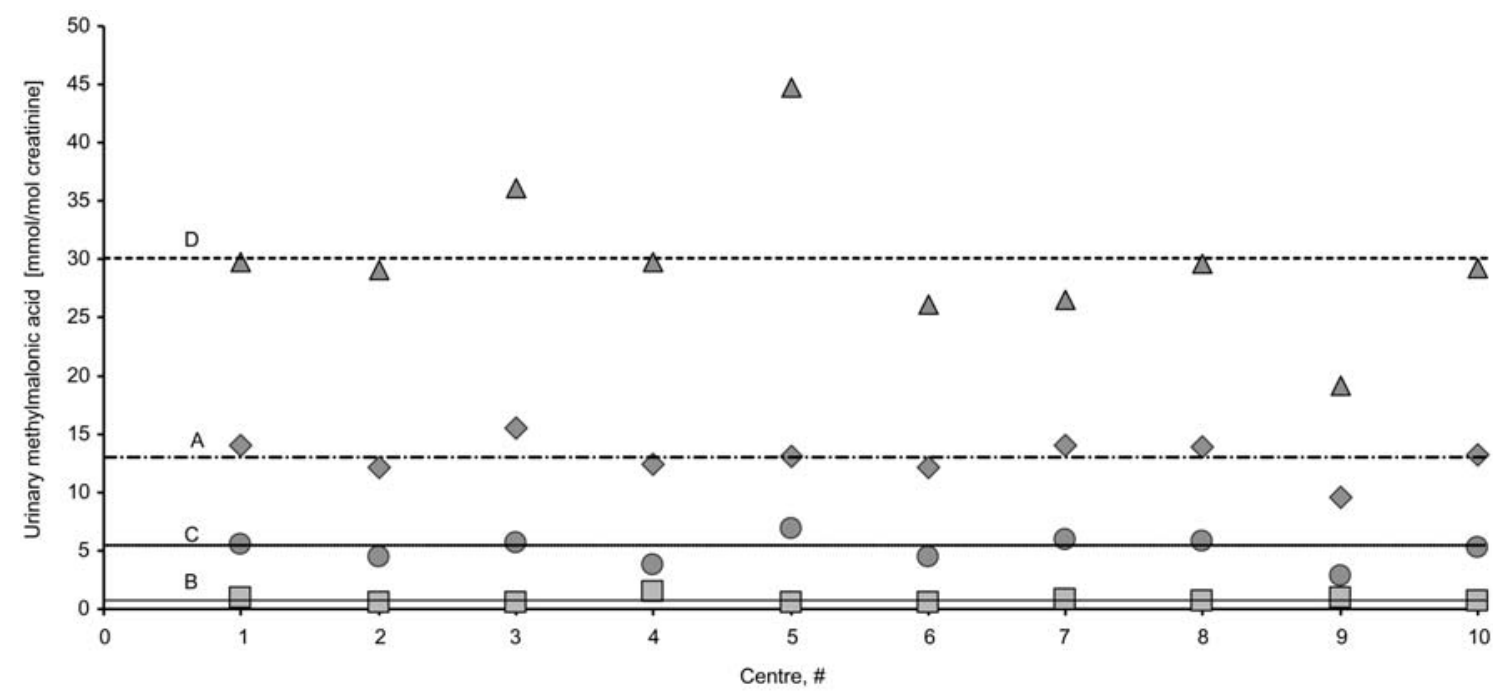

Figure 1 Individual urinary methylmalonic acid results reported from ten laboratories for four study samples. Sample A, rhomboids; sample B, squares; sample C, circles; sample D, triangles. Horizontal lines indicate the mean concentration reported for the four samples.

was correctly distinguished from urine with MMA concentrations typically found in cobalamin deficiency. However, the interlaboratory coefficients of variation observed in our investigation (approx. 20\% at the decision concentration level) are higher than typically found in proficiency testing programs for standard analytes and a deviation of $>20 \%$ from the mean concentration for samples was observed in a substantial proportion of measurements. Improvement in interlaboratory agreement of urinary MMA measurement might be achieved if materials for calibration and routine quality control of urinary MMA measurement become commercially available in the future.

In summary, our study has demonstrated the necessity of implementing quality assurance systems for urinary MMA measurement and the feasibility of an interlaboratory survey using lyophilised samples for this analyte was demonstrated. Implementation of a proficiency testing scheme for urinary MMA measurement in the future might be performed in accordance with the Guidelines of the European Cooperation for Accreditation (EA) (9).

\section{Acknowledgements}

The study was supported by the Hans-Fischer-Gesellschaft, Munich, Germany.

\section{References}

1. Klee GG. Cobalamin and folate evaluation: measurement of methylmalonic acid and homocysteine vs. vitamin B(12) and folate. Clin Chem 2000;46:1277-83.

2. Lindgren A. Elevated serum methylmalonic acid. How much comes from cobalamin deficiency and how much comes from the kidneys? Scand J Clin Lab Invest 2002; 62:15-20.

3. Moller J, Rasmussen K, Christensen L. External quality assessment of methylmalonic acid and total homocysteine. Clin Chem 1999;45:1536-42.

4. Marcell PD, Stabler SP, Podell ER, Allen RH. Quantitation of methylmalonic acid and other dicarboxylic acids in normal serum and urine using capillary gas chromatographymass spectrometry. Anal Biochem 1985;150:58-66.

5. Matchar DB, Feussner JR, Millington DS, Wilkinson RH Jr, Watson DJ, Gale D. Isotope-dilution assay for urinary methylmalonic acid in the diagnosis of vitamin B12 deficiency. A prospective clinical evaluation. Ann Intern Med 1987;106:707-10.

6. Rasmussen K, Nathan E. The clinical evaluation of cobalamin deficiency by determination of methylmalonic acid in serum or urine is not invalidated by the presence of heterozygous methylmalonic-acidaemia. J Clin Chem Clin Biochem 1990;28:419-21.

7. Magera MJ, Helgeson JK, Matern D, Rinaldo P. Methylmalonic acid measured in plasma and urine by stable-isotope dilution and electrospray tandem mass spectrometry. Clin Chem 2000;46:1804-10.

8. Rasmussen K, Moller J, Ostergaard K, Kristensen MO, Jensen J. Methylmalonic acid concentrations in serum of normal subjects: biological variability and effect of oral Lisoleucine loads before and after intramuscular administration of cobalamin. Clin Chem 1990;36:1295-9.

9. EA, European Co-operation for Accreditation: The ILAC G13 Guidelines for the Requirements for the Competence of Providers of Proficiency Testing Schemes. www. european-accreditation.org/Docs/0003_International/0001_ ILAC/00500_ILAC\%20G13\%202000.pdf. 